\title{
Medindo a constante dielétrica em líquidos: um estudo de caso para elaboração de uma proposta para formação de físicos experimentais
}

Measuring the dielectric constant in liquids: a case study to the creation of a proposal for the formation of experimental physicists

\author{
J. R. T. Reis ${ }^{1}$, A. G. Rodrigues ${ }^{2}$, N. M. Barbosa Neto*10 \\ ${ }^{1}$ Universidade Federal do Pará, Instituto de Ciências Exatas e Naturais, Programa de Pós-graduação em Física, Belém, PA, \\ Brasil \\ ${ }^{2}$ Universidade Federal do Pará, Instituto de Tecnologia, Programa de Curso de Nivelamento de Aprendizagem para Ciências \\ Básicas e Engenharias, Belém, PA, Brasil
}

Recebido em 19 de Março, 2018. Revisado em 05 de Junho, 2018. Aceito em 12 de Junho, 2018.

\begin{abstract}
Partindo de um problema clássico de livro texto de ensino superior (capacitor semipreenchido com dois dielétricos) propõem-se uma nova abordagem experimental que é empregada para a determinação da constante dielétrica estática de um líquido (hexano). Denominada de técnica do preenchimento do capacitor (TPC) esta consiste em medir a capacitância de um capacitor de placas paralelas com diferentes volumes de dielétrico e permite a eliminação de erros causados pela existência de capacitância parasita. Tendo sido o presente projeto elaborado a partir de um problema de livro texto, traçamos comentários sobre a possibilidade de que esta experiência, e as reflexões subjacentes, possam servir para a elaboração de uma proposta metodológica visando a formação de pesquisadores experimentais nas áreas de exatas (física, química e engenharias). Tal proposta é baseada em metodologias de aprendizagem por projetos e traz potencialidades para reformulação de cursos de física básica via uma maior integração entre teoria e prática. Ao final são feitas considerações a respeito das competências e habilidades desenvolvidas pelo estudante que participou da investigação.
\end{abstract}

Palavras-chave: Constante dielétrica, Método do preenchimento do capacitor, Aprendizagem baseada em projeto.

\begin{abstract}
Starting from a classical problem of a college textbook (the half-filled capacitor with two dielectrics), we propose a new experimental approach that is used to determine the static dielectric constant of a liquid (hexane). Called capacitor-filling technique, it consists in measuring the capacitance of a parallel plate capacitor with different volumes of dielectric and allows the elimination of errors caused by the parasitic capacitance. Since this project have been elaborated from a textbook problem, we have commented on the possibility that this experiment, and the underlying reflections, could serve to elaborate a methodological proposal aiming at the formation of experimental researchers in areas like physics, chemistry, and engineering. The proposal is supported on project-based learning methodology and brings potentialities for the reformulation of basic physics courses through the enhancement of the integration between theory and practice. Finally, considerations are made regarding the skills and competencies developed by the student who participated in the research.
\end{abstract}

Keywords: Dielectric constant, Capacitor filling technique, Project based learning

\section{Introdução}

Embora grande parte do nosso conhecimento em Física seja obtido a partir de investigações experimentais, devido a nossa cultura educacional os cursos introdutórios dessa ciência são, em geral, abordados de maneira teórica com base em metodologias expositivas com foco no que é ensinado, e não em práticas experimentais investigativas $[1,2]$. Em geral esta opção metodológica desestimula o interesse de uma parcela significativa dos alunos. E ainda pior, o contingente de alunos atraídos pela metodologia descrita acima (amplamente majoritária nas salas de

*Endereço de correspondência: newtonfisico@gmail.com aula, seja na educação básica ou no nível superior) parece estar diminuindo com o tempo. Dito de outra forma, um número cada vez maior de alunos parece se sentir enfadado pelas práticas tradicionais de ensino, quase que exclusivamente passivas e propedêuticas.

A baixa atratividade didática e motivacional dos cursos básicos de física certamente contribui para os índices de reprovação nessas disciplinas, o que, por sua vez, colabora para o aumento das taxas de evasão nos cursos de exatas. Não bastassem essas influências nada positivas para os indicadores acadêmicos dos cursos de graduação, a ênfase em uma abordagem meramente teórica contribui para o agravamento de um problema crítico para a ciência 
nacional que é a baixa densidade de pesquisadores experimentais, em especial na área de exatas. Portanto, para além de considerações de caráter didático-pedagógico, o desenvolvimento de metodologias que venham contribuir para a redução dos problemas citados é de grande relevância para as áreas de ensino e de educação em sentido amplo.

Neste trabalho apresenta-se brevemente a estrutura de um curso básico de eletromagnetismo, normalmente denominado em cursos de graduação como Física 3. Em seguida um pouco da física dos materiais dielétricos e o método experimental desenvolvido com o intuito de medir a constante dielétrica estática de líquidos, denominada no presente caso de técnica do preenchimento do capacitor (TPC), é discutido. Tendo sido o problema aqui exposto elaborado a partir da análise e discussão de uma questão retirada de um livro texto de física básica, traçamos comentários a respeito da possibilidade de se aprimorar este método de abordagem de problemas para servir como um protótipo para formação de pesquisadores experimentais nas áreas de exatas (física, química e engenharias). A abordagem proposta aqui foi testada com um estudante de graduação do terceiro semestre do curso de bacharelado em Física da Universidade Federal do Pará, que estava na época (primeiro semestre de 2015) cursando a disciplina de Física 3. As competências e habilidades percebidas na formação do aluno são descritas.

\section{Fundamentação teórica}

O conteúdo tradicional de cursos de eletromagnetismo básico envolve tópicos que vão desde conceitos como carga, força e campo (estáticos e variáveis no tempo) até o comportamento de campos na matéria [3-5]. Em um primeiro momento, os estudos relacionados às propriedades elétricas da matéria envolvem a ideia de dipolos elétricos e como estes interagem com campos estáticos e uniformes gerados por capacitores de placas paralelas. A partir da ideia de dipolo elétrico é possível obter o entendimento de propriedades simples de materiais dielétricos, tais como polarização, constante dielétrica dentre outras [3-5].

Materiais dielétricos são definidos como materiais isolantes que, quando submetidos a campos eletrostáticos externos, podem ser polarizados, ou seja, podem sofrer processos de separação de carga ou produzir alinhamento de dipolos elétricos já existentes. Líquidos polares e apolares, sem a presença de íons dispersos, são exemplos de materiais dielétricos bem como borracha, filmes poliméricos, vidros, entre outros [6]. A constante dielétrica estática é basicamente uma medida de quanto a presença de um dielétrico entre as placas de um capacitor aumenta sua capacidade de armazenar energia [3-5]. De uma forma bem simplista, podemos dizer que esse aumento na capacidade de armazenamento de energia em um capacitor, devido à presença de um dielétrico, ocorre por conta da formação, ou alinhamento, dos momentos de dipolo por unidade de volume na matéria.

É dentro desse contexto que é apresentado aqui um protótipo de metodologia para formação de físicos experimentais que pode ser empregada durante o transcorrer de um curso convencional de Física. A ideia central do método é a elaboração de projetos de experimentação a partir de problemas retirados de livros texto de Física em nível de graduação, ou de discussões realizadas em sala de aula. Uma das vantagens desse método é que neste o estudante trabalha com um nível de conhecimento mais em ressonância com o seu estágio de desenvolvimento intelectual no momento. Isto faz com que os níveis de abstração diminuam consideravelmente liberando esforço intelectual para ser usado no aprendizado do método científico experimental. Podemos organizar a metodologia proposta em três etapas apresentadas a seguir.

\subsection{Etapa 1: Organização dos tópicos e escolha dos problemas potenciais}

Esta etapa é semelhante à organização de um curso convencional de física, com a exceção de que o professor deve organizar um banco de prováveis questões com grande potencial para serem transformadas em projetos experimentais. Sugere-se que o professor parta de problemas do livro texto adotado a fim de facilitar a concepção do projeto, todavia, projetos experimentais também podem ser elaborados a partir da leitura de bibliografia complementar.

\subsection{Etapa 2: Análise e entendimento do problema escolhido}

Esta etapa consiste em discutir com o aluno, ou grupo de alunos, problemas do livro de modo a assegurar que os princípios básicos fundamentais para sua solução sejam bem assimilados. Para o presente trabalho o problema escolhido como motivação inicial foi um problema clássico sobre propriedades dielétricas de meios materiais, encontrado em praticamente todos os livros de física básica, [3-5] e que consiste em calcular a capacitância equivalente de um capacitor de placas paralelas semipreenchido, de forma simétrica, por dois dielétricos com constante dielétrica $K_{1}$ e $K_{2}$, veja Fig. 1.

A solução desse problema é bastante simples e pode ser facilmente entendida por alunos do curso básico de eletromagnetismo com conhecimento mínimo de álgebra

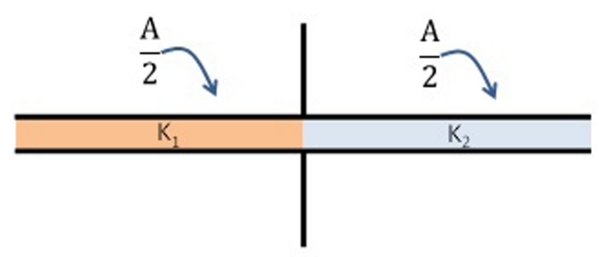

Figura 1: Esquema do problema motivador para o projeto experimental desenvolvido. Figura adaptada da referência [3]. 
elementar a das regras de associação de capacitores. Embora a solução deste seja bem conhecida, alguns detalhes são fornecidos aqui no sentido de auxiliar o leitor leigo na leitura do manuscrito. Da geometria da Fig. 1 percebe-se que o sistema é equivalente a dois capacitores em paralelo cuja capacitância equivalente é a soma de suas capacitâncias:

$$
\mathrm{C}_{\mathrm{eq}}=\mathrm{C}_{1}+\mathrm{C}_{2}
$$

Como a capacitância de um capacitor de placas paralelas, preenchido por um meio dielétrico é $\mathrm{C}=\frac{\mathrm{K} \varepsilon_{\mathrm{o}} \mathrm{A}}{\mathrm{d}}$, (sendo $\mathrm{K}$ a constante dielétrica; $\mathrm{A}$ área do capacitor; $\varepsilon_{\mathrm{o}}$ a permissividade do vácuo e d a distância entre as placas) temos que a capacitância equivalente para o problema em questão vale:

$$
\mathrm{C}_{\mathrm{eq}}=\frac{\mathrm{A} \varepsilon_{\mathrm{o}}}{2 \mathrm{~d}}\left(\mathrm{~K}_{1}+\mathrm{K}_{2}\right)
$$

Da Fig 1 nota-se que a área preenchida por cada dielétrico é metade da área das placas do capacitor. Uma vez apresentado o problema e o mesmo ser analisado pelo aluno ou grupo de alunos, passamos para a etapa 3 que é a generalização das condições do problema e sua execução como projeto experimental.

\subsection{Etapa 3: Generalização das condições problema e validação experimental do modelo}

Esta é a mais difícil, demorada e crítica fase do método proposto, todavia sua execução é fundamental para desenvolver no estudante as capacidades inerentes de um pesquisador experimental as quais podemos citar: 1) planejar um experimento científico, 2) coletar e analisar os dados experimentais, 3) correlacionar os resultados experimentais ao modelo teórico, 4) chegar a conclusões que validem, ou não, o modelo e 5) verificar possíveis consequências a partir das conclusões científicas obtidas. Os detalhes do procedimento experimental utilizado são apresentados na seção 3 .

Para o início desta etapa é fundamental o estímulo do estudante via questionamentos a respeito dos limites da solução canônica encontrada e como é possível realizar generalizações do problema. Esta fase também se caracteriza por fazer o estudante ir além do que está escrito nos livros textos criando ideias próprias a partir de discussões. No caso particular do presente problema, a partir da análise e discussão do tema, a pergunta feita ao estudante foi: existe uma forma geral para a capacitância equivalente que considere diferentes proporções de preenchimento entre os dielétricos $K_{1}$ e $K_{2}$ ? Em outras palavras: é possível escrevermos a capacitância equivalente como função do preenchimento do capacitor com quantidades arbitrárias de materiais dielétricos? A resposta é sim. Para isto devemos escrever a capacitância equivalente a partir do esquema mostrado na Fig. 2 , onde, por simplicidade, escolhemos placas quadradas para construção do modelo mais geral.

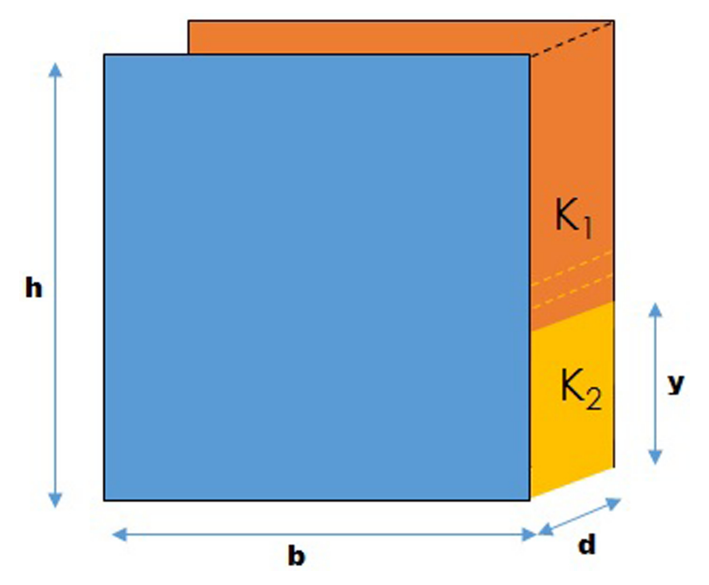

Figura 2: Esquema para generalização do problema inicial

Devido à presença dos diferentes dielétricos, o capacitor é subdividido em duas partes com as respectivas capacitâncias:

$$
\begin{aligned}
\mathrm{C}_{1} & =\frac{\mathrm{K}_{1} \varepsilon_{0} \mathrm{~A}_{1}}{\mathrm{~d}} \\
\mathrm{C}_{2} & =\frac{\mathrm{K}_{2} \varepsilon_{0} \mathrm{~A}_{2}}{\mathrm{~d}}
\end{aligned}
$$

Para geometria mostrada na Fig. 2 podemos escrever a capacitância equivalente como:

$$
\mathrm{C}_{\mathrm{eq}}=\mathrm{C}_{1}+\mathrm{C}_{2}=\frac{\varepsilon_{\mathrm{o}}}{\mathrm{d}}\left[\mathrm{K}_{1} \mathrm{~A}_{1}+\mathrm{K}_{2} \mathrm{~A}_{2}\right]
$$

a qual pode ser reescrita como:

$$
\mathrm{C}_{\mathrm{eq}}=\frac{\varepsilon_{\mathrm{o}} \mathrm{b}}{\mathrm{d}}\left[\mathrm{K}_{1} \mathrm{~h}+\left(\mathrm{K}_{2}-\mathrm{K}_{1}\right) \mathrm{y}\right]
$$

A equação (6) é uma expressão mais geral para o problema apresentado nos livros textos, onde tomando a variável $\mathrm{y}=\frac{\mathrm{h}}{2}$ obtêm-se como esperado a capacitância equivalente fornecida pela equação (2). Uma vez obtido a solução mais abrangente para o problema, podemos avançar para a realização de um experimento visando sua validação

No estudo de caso abordado a verificação experimental do modelo implica na construção de uma nova técnica para medida da constante dielétrica estática de líquidos, denominada TPC. Esta consiste em medir a capacitância, em um capacitor de placas paralelas com diferentes níveis de preenchimento por um líquido dielétrico. A partir da dependência linear verificada entre a capacitância e o volume de preenchimento, é possível, via correlação entre a curva experimental e a equação do modelo de preenchimento, obter o valor da constante dielétrica do líquido.

\section{Materiais e Métodos}

O aparato experimental consiste basicamente de um capacitor de placas paralelas, com área de 39,70 x 34,08 $\mathrm{mm}^{2}$ e distância de $0,97 \mathrm{~mm}$ entre estas, conectado a uma 
fonte de capacitância, modelo Fluke PM6304, montados de acordo com o esquema mostrado na Fig. 3(a) Os diferentes níveis de preenchimento com os dielétricos foram obtidos, empregando como dielétrico 1 o ar $\left(K_{1} \cong 1\right)$ e como dielétrico 2 um líquido com o qual o espaço entre as placas do capacitor é progressivamente preenchido, tal como ilustrado na Fig. 3(b). O líquido escolhido para realizar este experimento foi o hexano (marca ISOFAR), cuja constante dielétrica estática é bem determinada na literatura [7] e o grau de pureza é industrialmente garantido (98,5\%). Aqui é importante ressaltar que a água não foi utilizada como meio dielétrico, pois os experimentos realizados com esta indicam que mesmo água filtrada possui alto grau de impurezas químicas uma vez que a mesma é previamente tratada pelo sistema de abastecimento. Todas as medidas foram realizadas e temperatura ambiente.

\section{Resultados e Discussões}

Para implementação da TPC uma pequena modificação na equação (6) é realizada no sentido de reduzir o valor do erro experimental. A modificação feita é substituir a variável altura (y) pelo volume de preenchimento, o que é facilmente obtido uma vez que o volume preenchido entre as placas vale $\mathrm{V}=\mathrm{ybd}$, (veja geometria da Fig. 2). Realizando as substituições necessárias, e tomando como unitária a constante dielétrica do ar, podemos reescrever a equação (6) como:

$$
\mathrm{C}_{\mathrm{eq}}=\frac{\varepsilon_{\mathrm{o}} \mathrm{bh}}{\mathrm{d}}+\left[\frac{\varepsilon_{\mathrm{o}}\left(\mathrm{K}_{\mathrm{l}}-1\right)}{\mathrm{d}^{2}}\right] \mathrm{V}
$$

De posse da equação (7), que basicamente é a equação (6) do modelo geral reescrita para colocar a capacitância equivalente do sistema em função do volume preenchido pelo líquido, realiza-se o experimento utilizando hexano como líquido dielétrico. A determinação do volume de dielétrico inserido é facilmente obtida através do uso de uma seringa graduada. Na Fig. 4 um resultado típico obtido pela aplicação da TPC para o hexano é mostrado.

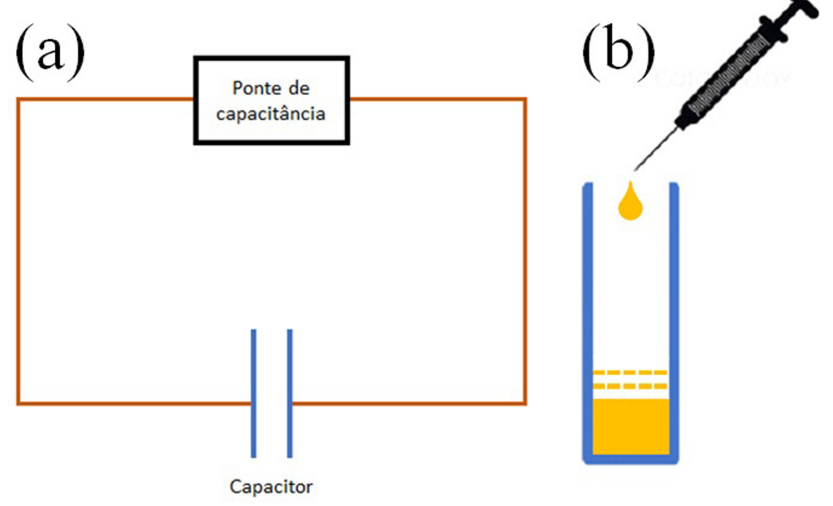

Figura 3: (a) Esquema experimental utilizado para medidas de constante dielétrica e (b) procedimento de preenchimento do capacitor com um líquido dielétrico.

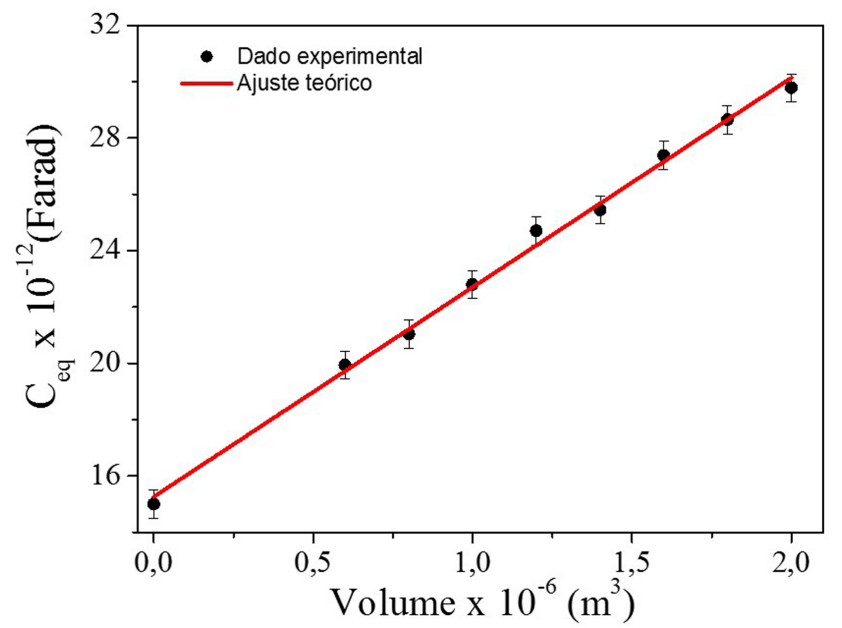

Figura 4: Resultado obtido para o experimento do preenchimento do capacitor utilizando como líquido dielétrico o hexano. Os círculos sólidos são os pontos experimentais e a linha vermelha sólida corresponde ao ajuste linear realizado.

Observa-se, como esperado da equação (7), que a capacitância equivalente tem uma dependência linear com o volume do preenchimento. Além disso, uma vez que as grandezas como distância entre as placas e permissividade do vácuo $\left(\varepsilon_{\mathrm{o}}\right)$ são constantes conhecidas pode-se obter o valor da constante dielétrica do líquido igualando-se o valor do coeficiente angular obtido do ajuste da curva experimental com o termo $\left[\frac{\varepsilon_{\mathrm{o}}\left(\mathrm{K}_{1}-1\right)}{\mathrm{d}^{2}}\right]$, obtido a partir do modelo teórico, equação (7). Realizando tal procedimento obtém-se um valor de 1,82 para a constante dielétrica do hexano, valor este que difere em menos de $5 \%$ do valor encontrado na literatura $(1,90)[7]$.

Por fim, outra vantagem percebida ao longo do estudo é que a TPC resolve um problema crítico existente em medidas de constante dielétrica que é o problema da capacitância parasita. [6,8] Este problema é causado por possíveis diferenças de impedância existente ao longo do circuito usado para medir a constante dielétrica que criam uma capacitância aditiva no valor medido. Tal fato representa uma grande fonte de erro nas medidas de constante dielétrica que utilizam o método convencional. Isto ocorre, pois no procedimento convencional a constante dielétrica é obtida a partir da razão entre as capacitâncias medidas com o capacitor preenchido e o capacitor vazio. Denominando de $\mathrm{C}_{\mathrm{v}}$ e $\mathrm{C}_{\mathrm{p}}$ as capacitâncias obtidas, com o capacitor vazio e preenchido respectivamente, temos:

$$
\begin{gathered}
\mathrm{C}_{\mathrm{v}}=\frac{\varepsilon_{0} \mathrm{~A}}{\mathrm{~d}} \\
\mathrm{C}_{\mathrm{P}}=\frac{\mathrm{K} \varepsilon_{0} \mathrm{~A}}{\mathrm{~d}}
\end{gathered}
$$

Tomando as razões das equações (8) e (9), temos:

$$
\mathrm{K}=\frac{\mathrm{C}_{\mathrm{P}}}{\mathrm{C}_{\mathrm{V}}}
$$

O problema surge, pois, uma vez que a capacitância parasita é aditiva a mesma não será eliminada pela razão 
tomada na equação 10 , o que implica no aparecimento de erro na medida da constante dielétrica. Ao contrário do método tradicional, a TPC resolve esse problema de forma bastante elegante. Uma vez que a capacitância parasita existente é aditiva podemos reescrever a equação (5) como:

$$
\begin{gathered}
\mathrm{C}_{\mathrm{eq}}=\mathrm{C}_{1}+\mathrm{C}_{2}+C_{P}=\frac{\varepsilon_{\mathrm{o}}}{\mathrm{d}}\left[\mathrm{K}_{1} \mathrm{~A}_{1}+\mathrm{K}_{2} \mathrm{~A}_{2}\right]+C_{P} \\
\mathrm{C}_{\mathrm{eq}}=\frac{\varepsilon_{\mathrm{o}} \mathrm{b}}{\mathrm{d}}\left[\mathrm{K}_{1} \mathrm{~h}+\left(\mathrm{K}_{2}-\mathrm{K}_{1}\right) \mathrm{y}\right]+\mathrm{C}_{\mathrm{P}}
\end{gathered}
$$

Reescrevendo a equação 12 para ter como variável o volume de preenchimento obtêm-se

$$
\mathrm{C}_{\mathrm{eq}}=\left[\frac{\varepsilon_{\mathrm{o}} \mathrm{bh}}{\mathrm{d}}+\mathrm{C}_{\mathrm{p}}\right]+\left[\frac{\varepsilon_{\mathrm{o}}\left(\mathrm{K}_{\mathrm{l}}-1\right)}{\mathrm{d}^{2}}\right] \mathrm{V}
$$

Fica claro da equação (13) que no método aqui desenvolvido a capacitância parasita contribui apenas no coeficiente linear obtido dos dados experimentais não influenciando no coeficiente angular do mesmo, de onde obtemos o valor da constante dielétrica.

Por fim, verificou-se que o estudante do segundo ano de graduação do curso de bacharelado em Física, seguindo as etapas da metodologia proposta, foi capaz de elaborar e executar um experimento com o objetivo de verificar a generalização de um problema teórico do livro texto. Como consequência das discussões realizadas no presente trabalho o estudante foi capaz de perceber que a TPC pode ser usada para resolver um problema tradicional nas medições de constantes dielétricas estáticas, que é o problema da capacitância parasita. Outro aspecto importante é que o mesmo após o termino formal do curso de física 3, se manteve engajado em projetos de iniciação cientifica e hoje cursa mestrado em Física na Universidade Federal do Pará. Tal fato é um indicativo do possível impacto da metodologia apresentada, que é uma vertente das metodologias de aprendizagem baseadas em projetos, sobre a motivação do estudante para realização de atividades profissionais em ciência experimental.

\section{Conclusões}

No presente trabalho, em um curso convencional de física e utilizando um problema sobre capacitores e propriedades dielétricas de materiais trabalhou-se com o aluno a abordagem teórica do problema, a generalização do mesmo e a posterior construção do experimento com coleta e análise de dados. Foi observado que o estudante do segundo ano do curso de física, sob a supervisão do professor, foi capaz de, partindo dos princípios básicos relacionados ao tema, generalizar um problema teórico e elaborar e executar o experimento correspondente. É também observado que além dos já citados fatos o estudante foi capaz de perceber que a técnica experimental desenvolvida pode ser usada para resolver um problema existente nas medições de constantes dielétricas estáticas (capacitância parasita), tendo esta descoberta impactos na própria área de propriedades dielétricas de materiais. Além disso, observamos que o mesmo foi capaz de, ao longo do processo, absorver as principais características do método experimental tais como procedimento por comparação, tentativa e erro, observação do fenômeno, correlação dos dados com o modelo teórico entre outros.

Baseado no exposto, o estudo de caso aqui apresentado nos instiga a propor a presente abordagem como um protótipo para a elaboração de um método de ensino da física experimental bem como para o de uma nova estrutura curricular das disciplinas científicas básicas. Nesse sentido, precisamos, em futuro próximo, estabelecer critérios metodológicos mais rigorosos e aprofundar o embasamento teórico do método proposto (características do problema; da orientação e de formas de avaliação) a fim de fornecer subsídios para novas práticas. Além disso, embora tenha sido executado com um problema de eletromagnetismo é perfeitamente factível estender o presente método para outras áreas da ciência. Vale também ressaltar que os autores acreditam que esta abordagem pode perfeitamente ser aplicada não só em cursos universitários, mas em escolas de nível médio como uma alternativa para elaboração de trabalhos científicos.

\section{Agradecimentos}

Os autores são gratos ao CNPq, FAPESPA e CAPES bem como a Pró-reitoria de extensão da Universidade Federal do Pará pelo apoio dado a esta pesquisa. Os autores também estão em dívida com o Prof. Sanclayton Geraldo Carneiro Moreira do Programa de Pós-graduação em Física da Universidade Federal do Pará pelo uso das instalações experimentais e pelo auxílio no uso da ponte de capacitância.

\section{Referências}

[1] J.P. Alves Filho, Atividade experimental: do método à prática construtivista. Tese de Doutorado, Universidade Federal de Santa Catarina, Florianópolis (2000).

[2] N.A. Grandini e C.R. Grandini, Rev. Bras. Ens. Fis. 26, 251 (2004).

[3] D. Halliday e R. Resnick, Física (Livros Técnicos e Científicos, Rio de Janeiro, 1984), v. 3.

[4] H.M. Nussenzveig, Curso de Física Básica (Editora Edgard Blücher, São Paulo, 1997), v. 3.

[5] E.M. Purcel, Curso de Física de Berkeley: Eletricidade e Magnetismo (Editora Edgard Blücher, São Paulo, 1973), v. 2.

[6] O. Gallot-Lavallèe, Dielectric materials and electrostatics, (Wiley-ISTE, Hoboken, 2013).

[7] Z. Hu, A.P. Willard, R.J. Ono, C.W. Bielawski, P.J. Rossky e D.A. Vanden Bout, Nat. Commun. 6, 8246 (2015).

[8] https://en.wikipedia.org/wiki/Parasitic_ capacitance, acessado em 22/01/2018. 